\title{
Studies of Gardiovascular Responses to Some Endogenous Pressor and Hypotensive Agents in Conscious Stroke-Prone Spontaneously Hypertensive Rats of Different Ages
}

\author{
Seijiro Hara, M.A., Mitsuo Matsumura, Ph.D., \\ and Motohiko UedA, Ph.D.
}

\begin{abstract}
SUMmary
The effects of the endogenous pressor agents noradrenaline (NA), and angiotensin II (Ang II), and of the hypotensive agents acetylcholine (ACh) and adenosine (ADS), on blood pressure and heart rate in conscious and unrestrained stroke-prone spontaneously hypertensive rats (SHR-SP) and normotensive Wistar Kyoto rats (WKY) of different ages (4-9 weeks old) were investigated. Pressor responses to NA were enhanced in 7- and 9- week-old SHR-SP compared with those in WKY, but pressor responses to Ang II in SHR-SP were not different from those in WKY at all ages. The bradycardias following pressor responses to NA and Ang II were markedly attenuated in SHR-SP, especially older ones. Hypotensive responses to ACh were enhanced in SHR-SP, particularly at 9 weeks of age. However, hypotensive responses to ADS were attenuated in SHR-SP, especially at 7 weeks of age. Transient fall of heart rate due to $\mathrm{ADS}$ was also attenuated in 7- and 9- week-old SHR-SP. These alterations of hemodynamic or cardiovascular responses in SHR-SP became more evident in the established stages of hypertension.

These results suggest intimate relationships of the enhanced pressor responses to NA, attenuated bradycardias following pressor effects with NA or Ang II, and the attenuated hypotensive responses to ADS with the development or the maintenance of hypertension in SHR-SP. (Jpn Heart J 34: 439449,1993 )
\end{abstract}

Key Words:

SHR-SP Blood pressure Noradrenaline Acetylcholine Adenosine

Q TROKE-PRONE spontaneously hypertensive rats (SHR-SP) established $\checkmark$ from SHR ${ }^{1)}$ have been used to elucidate the mechanisms of essential hypertension. Many investigators have reported enhanced vascular constriction in re-

From Shionogi Research Laboratories, Shionogi \& Co., Ltd., Fukushima-ku, Osaka 553, Japan Address for correspondence: Motohiko Ueda, Ph.D., Shionogi Research Laboratories, Shionogi \& Co, Ltd., Fukushima-ku, Osaka 553, Japan.

This work was previously presented in abstract form (Jpn. Heart J. 30: 550, 1989).

Received for publication September 4, 1992.

Accepted February 18, 1993. 
sponse to endogenous vasoactive agents such as noradrenaline (NA) and angiotensin II (Ang II $)^{2-4)}$ or diminished vasodilation in response to endogenous vasoactive agents such as acetylcholine $(\mathrm{ACh})^{5-7)}$ in SHR-SP using isolated or in situ arterial preparations. These researchers suggested that such abnormal vascular reactivities might contribute to the development or maintenance of hypertension in SHR-SP. Despite investigations focusing on the reactivities of blood vessels, study of the effects of endogenous vasoactive agents on blood pressure and heart rate in whole animals, especially in the conscious state, has only been done with SHR $^{8-13)}$ and not with SHR-SP. As SHR-SP demonstrate a more rapid rise of blood pressure than SHR and an increased incidence of stroke, it is expected that more interesting cardiovascular effects of endogenous vasoactive agents might be observed in SHR-SP.

In the present study, we examined the effects of several endogenous pressor (NA and Ang II) and hypotensive agents (ACh and adenosine (ADS)) on blood pressure and heart rate in 4 to 9 week old conscious and unrestrained SHR-SP and compared these responses with those in WKY.

\section{Methods}

Animals: Male SHR-SP and WKY were obtained from colonies maintained at Aburahi Laboratories (Shionogi \& Co., Ltd.). These rats were maintained on CAl rat chow (Clea Japan; $0.24 \%$ sodium, 25.6\% protein) until 6 weeks old and thereafter on SP rat chow (Funabashi; $0.39 \%$ sodium, $20.8 \%$ protein) ${ }^{14}$.

Recording of blood pressure and heart rate in conscious rats: Rats 4, 5, 7 and 9 weeks old were anesthetized with sodium pentobarbital (60 $\mathrm{mg} / \mathrm{kg}$, i.p.), and a polyethylene catheter (SP10, SP3I or SP45; Natsume) was inserted into the right femoral artery and vein. The SP10 joined to SP45 by fusing (SP10+SP45) was used as the arterial catheter for rats 4, 5 and 7 weeks old. SP31 was used as the arterial catheter for rats 9 weeks old. The venous catheters used were SP10+SP45 for rats 4 weeks old, SP10+SP45 or SP31 for rats 5 and 7 weeks old and SP45 for rats 9 weeks old. The arterial and venous catheters, filled with heparinized physiological saline, were inserted into the respective arteries and veins and were exteriorized subcutaneously at the back of the neck. After the surgery, rats werc houscd in individual plastic cages and intravenously injected with heparinized physiological saline $(140 \mathrm{U} / \mathrm{ml}, 0.2 \mathrm{ml})$ twice a day, before being subjected to the experiments.

Two to four days after surgery, the rats were placed in a small plastic cage (8 $(\mathrm{L}) \times 19(\mathrm{~W}) \times 12.5(\mathrm{H}) \mathrm{cm})$ containing wooden chips. Experiments were performed with conscious and unrestrained rats. Arterial blood pressure was measured using a pressure transducer (MPU-0.5-290-0-III, TP-200T; Nihon 
Kohden) and medical recorder (PMP-3004; Nihon Kohden) or carrier amplifier (AP-620G; Nihon Kohden) with a pen recorder (WT-645G; Nihon Kohden). Mean arterial pressure (MAP) was calculated as follows: diastolic pressure $+1 / 3$ (systolic-diastolic pressure). Heart rate (HR) was determined using a cardiotachometer (Shionogi Research Laboratories) triggered by pulse pressure signals. Since MAP and HR easily fluctuated with the spontaneous movements of rats, they were measured when the rats were in a resting condition. To determine the effects of drugs on MAP and HR, they were administered intravenously via the inserted venous catheter.

Drugs: Drugs used in the present experiments were as follows: dl-noradrenaline (NA, Sankyo), angiotensin II (Ang II, Peptide Institute), acetylcholine (ACh, Daiichi Seiyaku), adenosine (ADS, Kohjin). All drugs were dissolved or dilutcd with physiological saline and were intravenously injected at a volume of 1 $\mathrm{m} l / \mathrm{kg}$.

Statistical analysis: Data are expressed as the meantstandard error. Statistical significance among the eight groups (four different age groups for each rat strain) was evaluated by Tukey's method after one-way analysis of variance.

\section{Results}

Mean arterial pressure (MAP), heart rate (HR) and body weight (BW) in SHR-SP and WKY: MAP in SHR-SP increased markedly from 4 weeks $(4 \mathrm{~W})$ to $9 \mathrm{~W}$ but MAP in WKY showed only mild increases. At all ages except $4 \mathrm{~W}, \mathrm{MAP}$ in SHR-SP was significantly higher than that in WKY (Table I). HR decreased with aging in both strains and no differences were observed between SHR-SP and WKY at any age (Table I). BW also did not differ between

Table I. Body Weight (BW), Mean Arterial Blood Pressure (MAP) and Heart Rate (HR) in Conscious and Unrestrained SHR-SP and WKY at Different Ages

\begin{tabular}{|c|c|c|c|c|c|}
\hline Strain & $\begin{array}{c}\text { Age } \\
\text { (wecks) }\end{array}$ & $\mathrm{n}$ & $\begin{array}{l}\text { BW } \\
\text { (g) }\end{array}$ & $\begin{array}{c}\text { MAP } \\
(\mathrm{mmHg})\end{array}$ & $\begin{array}{c}\text { HR } \\
\text { (beats/min) }\end{array}$ \\
\hline \multirow[t]{4}{*}{ SHR-SP } & 4 & 6 & $61 \pm 3$ & $100 \pm 3$ & $382 \pm 2$ \\
\hline & 5 & 13 & $98 \pm 2$ & $119 \pm 4^{a, c}$ & $366 \pm 8$ \\
\hline & 7 & 10 & $164 \pm 4^{c, c}$ & $156 \pm 4^{a, c, c}$ & $335 \pm 5^{b}$ \\
\hline & 9 & 8 & $223 \pm 5^{c, c}$ & $176 \pm 4^{a, c, c}$ & $317 \pm 5^{c, p}$ \\
\hline \multirow[t]{4}{*}{ WKY } & 4 & 6 & $60 \pm 2$ & $83 \pm 3$ & $375 \pm 8$ \\
\hline & 5 & 11 & $98 \pm 4$ & $98 \pm 2$ & $342 \pm 12$ \\
\hline & 7 & 9 & $170 \pm 4^{c, c}$ & $105 \pm 1^{c}$ & $304 \pm 10^{\mathrm{c}, \mathrm{d}}$ \\
\hline & 9 & 8 & $223 \pm 7 c$ & $117 \pm 4$ & $284 \pm 8$ \\
\hline
\end{tabular}

Significant differences were evaluated by Tukey's method after one-way analysis of variance. a=significantly different from the age-matched WKY $(\mathrm{P}<0.01)$;

$b, c=$ significantly different from 4 -week old rats $(P<0.05, P<0.01)$;

$\mathrm{d}, \mathrm{e}=$ significantly different from 5 -week old rats $(\mathbf{P}<0.05, \mathbf{P}<0.01)$. 


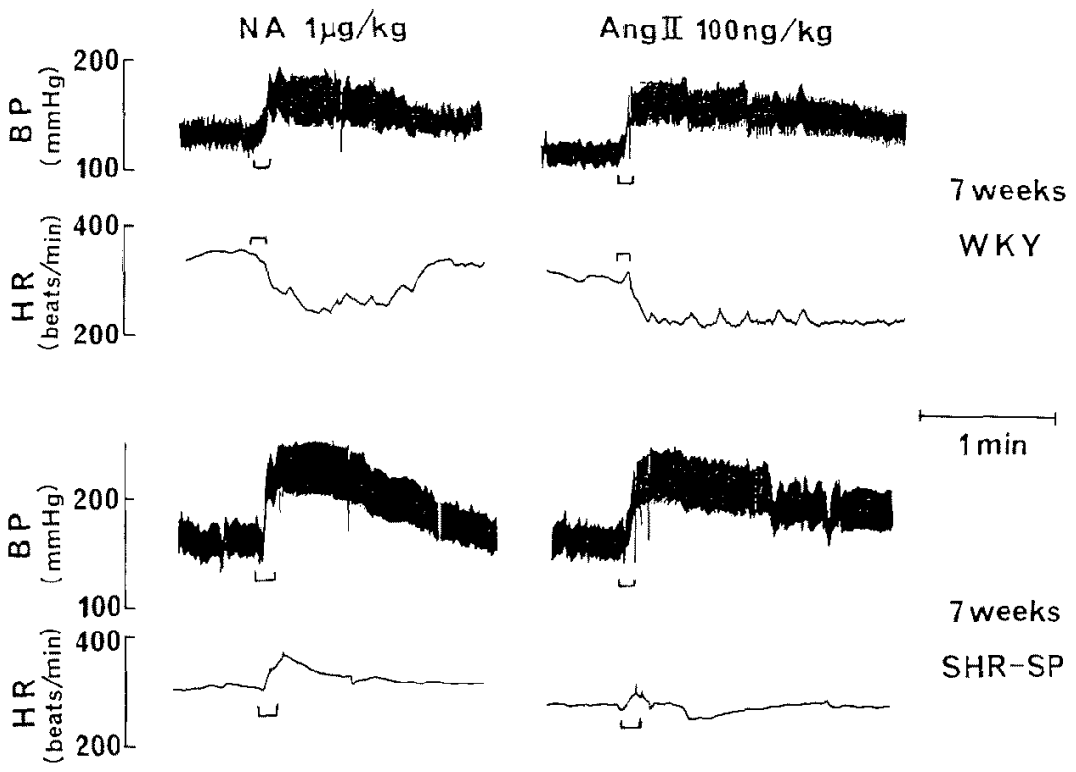

Fig. 1. Typical recordings of the effects of intravenous injection of noradrenaline (NA) and angiotensin II (Ang II) on blood pressure (BP) and heart rate (HR) in conscious and unrestrained 7 -week-old SHR-SP and WKY. Almost no bradycardic responses in SHR-SP were observed on administration of NA and Ang II.

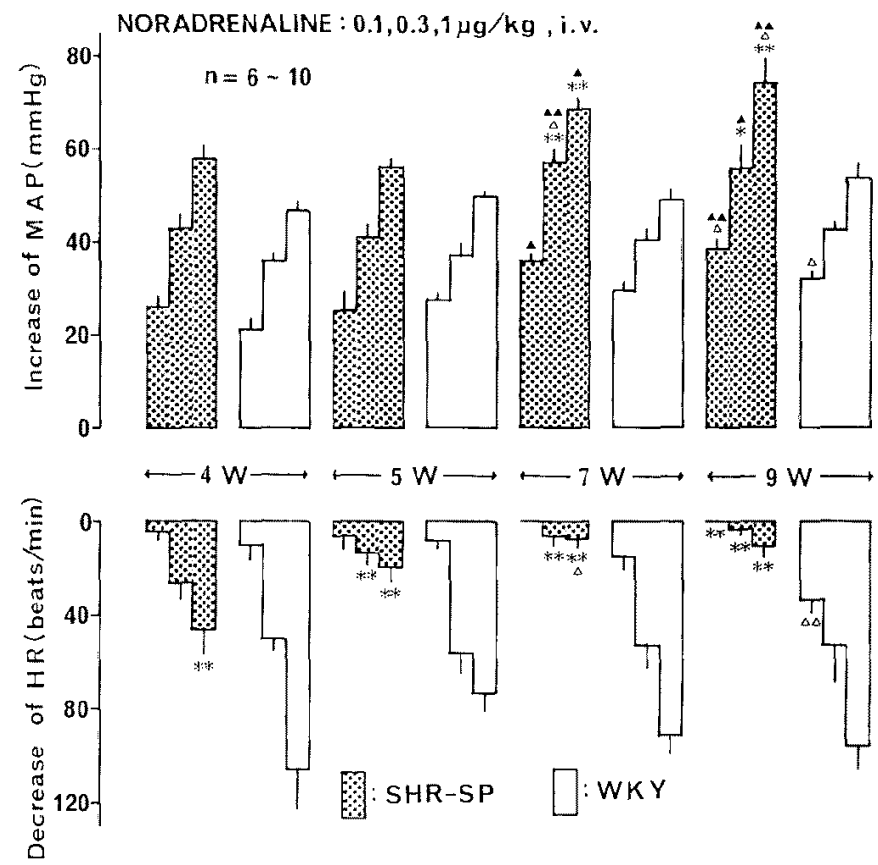

Fig. 2. Effects of intravenous injection of noradrenaline on mean arterial pressure (MAP) and heart rate (HR) in conscious and unrestrained SHR-SP and WKY of different ages. Dose-response relationships are shown in the staircase column with standard error bars. Significant differences were evaluated by Tukey's method after one-way analysis of variance.

****: Significantly different from the age-matched WKY $(\mathrm{P}<0.05, \mathrm{P}<0.01) ; \quad \triangle, \triangle \triangle$ : Significantly different from the 4-week-old rats $(\mathrm{P}<0.05, \mathbf{P}<0.01)$; $\quad \mathbf{\Delta}, \mathbf{A}$ : Significantly different from the 5-week-old rats $(\mathrm{P}<0.05, \mathrm{P}<0.01)$. 


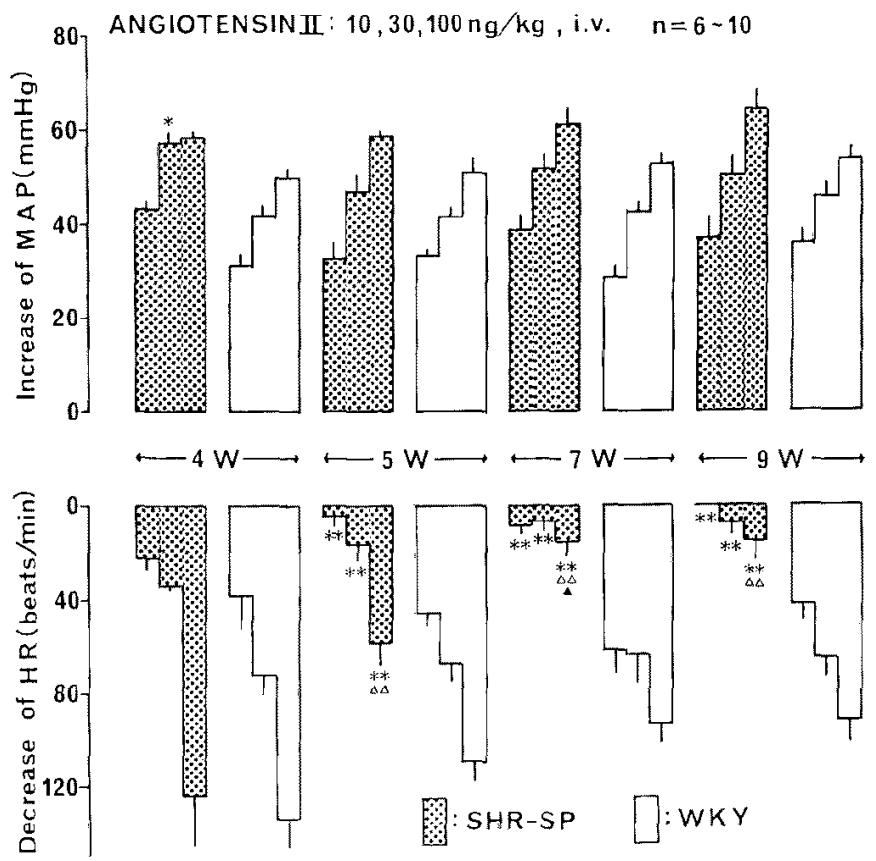

Fig. 3. Effects of intravenous injection of angiotensin II on mean arterial pressure (MAP) and hcart rate (HR) in conscious and unrestrained SHR-SP and WKY of different ages. Dose-response relationships are shown as in Fig. 2. Significant differences were evaluated as for Fig. 2.

***: Significantly different from the age-matched WKY $(P<0.05, P<001)$; $\triangle \triangle$ : Significantly different from the 4 -week-old rats $(\mathbf{P}<0.01) ; \quad \Delta$ : Significantly different from the 5-week-old rats $(\mathrm{P}<0.05)$.

\section{SHR-SP and WKY.}

Effects of pressor agents: Typical recordings of the effects of NA and Ang II on MAP and HR are shown in Figure 1. Intravenous injection of NA $(0.1-1 \mu \mathrm{g} / \mathrm{kg})$ caused dose-dependent increases of MAP and decreases of HR. The pressor effects of NA in SHR-SP at 7 and $9 \mathrm{~W}$ of age were greater than those in age-matched WKY, and also greater than those in SHR-SP at 4 and 5 $W$ of age (Fig. 2). Almost no changes in the pressor effects of NA in WKY were observed with aging except at $9 \mathrm{~W}$, when the smallest dose of NA caused a significantly greater response than at $4 \mathrm{~W}$. The bradycardias following pressor responses with NA were markedly attenuated in SHR-SP, especially at advanced ages (Fig. 1, 2).

Ang II (10-100 ng/kg, i.v.) also caused dose-dependent increases of MAP and decreases of HR. The pressor effects of Ang II in SHR-SP were similar to those in age-matched WKY except at $4 \mathrm{~W}$, when a more potent pressor effect was observed in SHR-SP at $30 \mathrm{ng} / \mathrm{kg}$, i.v. (Fig. 3). The pressor effects of Ang II 


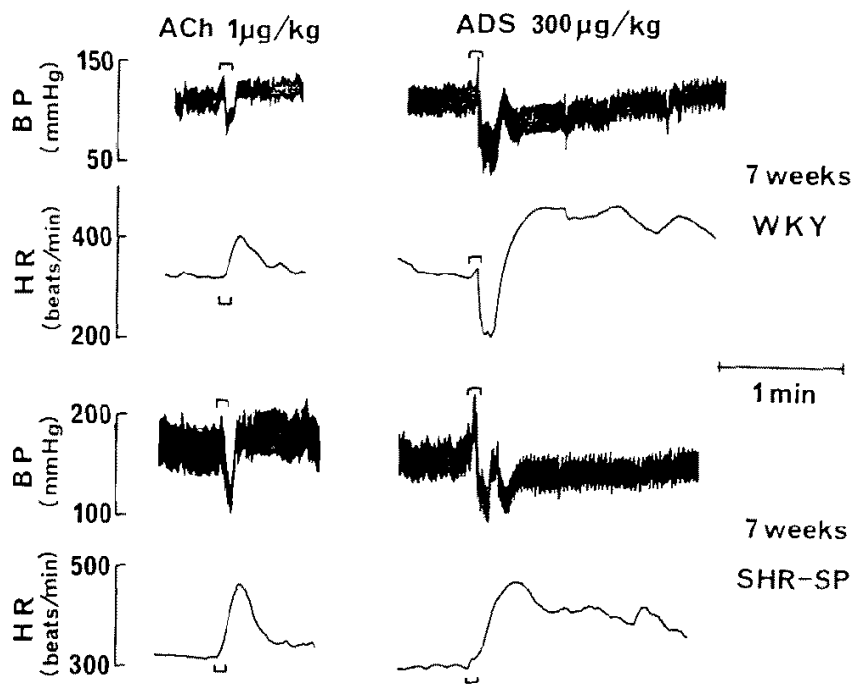

Fig. 4. Typical recordings of the effects of intravenous injection of acetylcholine $(A C h)$ and adenosine (ADS) on blood pressure (BP) and heart rate (HR) in conscious and unrestrained 7-week-old SHR-SP and WKY. No negative chronotropic effect with ADS was observed in SHR.SP.



Fig. 5. Hypotensive effects of intravenously injected acetylcholine and adenosine in conscious and unrestrained SHR-SP and WKY of different ages. Dose-response relationships are shown as in Fig. 2. Significant differences were evaluated as for Fig. 2. Data in 5-week-old animals were similar to those in 4-week- old animals (not shown).

***: Significantly different from the age-matched WKY $(P<0.05, P<0.01) ; \quad \triangle, \triangle \Delta$ : Significantly different from the 4-week-old rats $(\mathrm{P}<0.05, \mathrm{P}<0.01)$. 

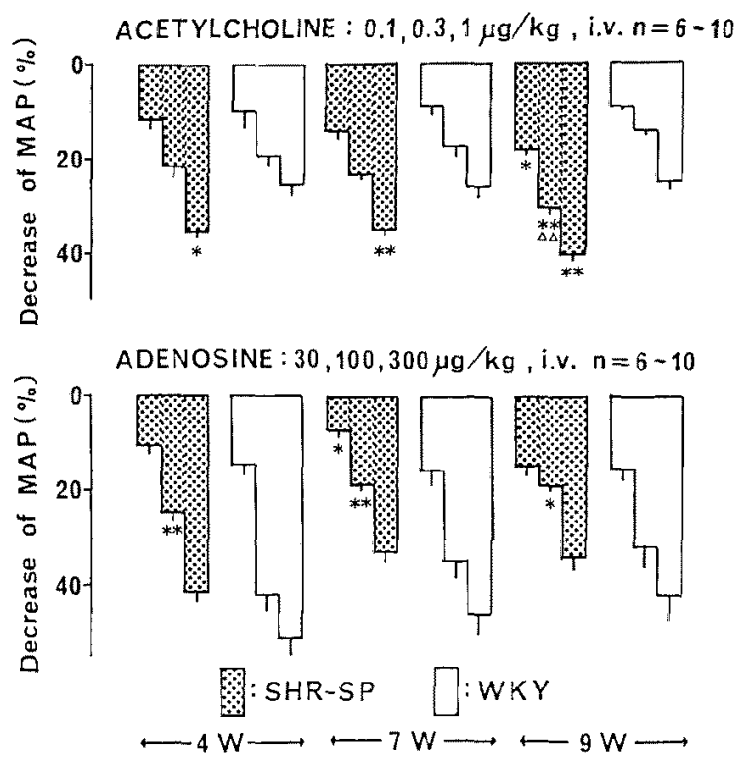

Fig. 6. Percent decreases in mean arterial pressure (MAP) with intravenously injected acetylcholine and adenosine in conscious and unrestrained SHR-SP and WKY of different ages. Dose-response relationships are shown as in Fig. 2. Significant differences were evaluated as for Fig. 2. Data in 5-week-old animals were similar to those in 4-week-old animals (not shown).

*,**: Significantly different from the age-matched WKY $(\mathbf{P}<0.05, \mathbf{P}<0.01)$; $\triangle \triangle$ : Significantly different from the 4 -week-old rats $(\mathrm{P}<0.01)$.

in both strains did not change with aging. However, the bradycardia following pressor responses with Ang II was attenuated in SHR-SP at 5, 7 and $9 \mathrm{~W}$, and this attenuation progressed with aging (Fig. 3).

Effects of hypotensive agents: Typical recordings of the effects of $\mathrm{ACh}$ and $\mathrm{ADS}$ on MAP and $\mathrm{HR}$ are shown in Figure 4. Intravenous injection of ACh $(0.1-1 \mu \mathrm{g} / \mathrm{kg})$ caused dose-dependent decreases in MAP and increases in HR. Decreases in HR with ACh were not observed at any age in SHR-SP and WKY. Absolute hypotensive responses to ACh in SHR-SP were greater than those in WKY at all ages and increased with aging (Fig. 5). The percent decreases in MAP with ACh were also greater in SHR-SP than those in WKY at 4, 7 and $9 \mathrm{~W}$ (Fig. 6). The percent decreases in MAP with ACh did not change with aging in WKY, but wcre greater in $9 \mathrm{~W}$ than in 4 and $5 \mathrm{~W}$ SHR-SP. Increases in HR following hypotensive response in SHR-SP were similar to those of agc-matched WKY at all ages (data not shown).

Intravenous injection of ADS (30-300 $\mathrm{ng} / \mathrm{kg}$ ) caused dose-dependent decreases in MAP and transient decreases followed by increases in HR. Absolute hypotensive responses to ADS in SHR-SP did not differ from those in WKY at 


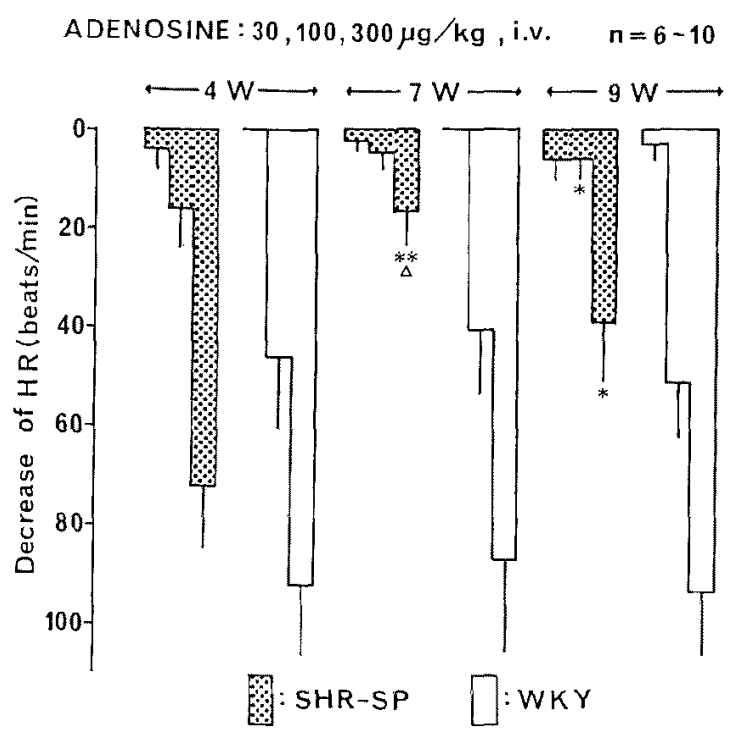

Fig. 7. Transient decreases in heart rate (HR) by intravenously injected adenosine in conscious and unrestrained SHR-SP and WKY of different ages. Doseresponse relationships are shown as in Fig. 2. Significant differences were evaluated as for Fig. 2. Data in 5-week-old animals were similar to those in 4-week- old animals (not shown).

*,**: Significantly different from the age-matched WKY $(\mathbf{P}<0.05, \mathbf{P}<0.01)$; $\triangle$ : Significantly different from the 4-week-old rats $(P<0.05)$.

any age, and did not change with aging, except at $9 \mathrm{~W}$ when the smallest dose of ADS caused a significantly greater response than at $4 \mathrm{~W}$ (Fig. 5). However, the percent decreases in MAP with ADS were attenuated in SHR-SP at 4, 7 and 9 W, and in both strains, no changes with aging were observed (Fig. 6). Transient decreases in HR in SHR-SP were smaller than those in WKY at 7 and $9 \mathrm{~W}$ (Fig. 7), however, tachycardic responses in SHR-SP were similar to those of agematched WKY of all ages (data not shown).

\section{Discussion}

In this study, we examined the effects of some endogenous vasoactive agents on blood pressure and heart rate in conscious and unrestrained SHR-SP and WKY in order to investigate the effects of these agents under normal physiological conditions.

Pressor responses to NA in SHR-SP were not different from those in WKY at 4 and $5 \mathrm{~W}$, but were significantly greater than those in WKY at 7 and $9 \mathrm{~W}$. On the other hand, pressor responses to Ang II were not different between SHRSP and WKY at any age. These results show that pressor responses to NA in SHR-SP were specifically enhanced with aging. In conscious SHR, pressor re- 
sponses to NA were also reported to have been enhanced ${ }^{8}$. However, other investigators showed that the pressor responses to both NA and Ang II in SHR were not different from those in WKY in the intact conscious state. ${ }^{9}$ For the vascular reactivities in SHR-SP, several investigators showed enhanced vasoconstriction following both NA and Ang $\mathrm{II}^{2-4)}$. Thus, our result, which shows specific enhancement of the pressor effect of NA, can not be fully explained by the previously reported vascular reactivities in SHR-SP. Our results may suggest that the vascular reactivity to NA but not to Ang II in the resistance vessels, which really determines systemic blood pressure, is enhanced, and it might be related to the enhancement of $\alpha$-adrenergic responses or reduction of $\beta$-adrenergic responses. ${ }^{15)}$

Bradycardias induced by both NA and Ang II were attenuated in SHR-SP, especially in older ones. Attenuation of the baroreceptor reflex has been widely reported in several hypertensive model animals and hypertensive patients. ${ }^{(6)-21)}$ Furthermore, this attenuation has been suggested to be secondary to hypertension. ${ }^{18), 19)}$ Our results on the attenuation of reflex bradycardia in SHR-SP seemed to correlate with these previous findings, because the attenuation of reflex bradycardia in SHR-SP became clearer with aging. It is noteworthy that the reflex bradycardia following NA administration in SHR-SP was attenuated even in $4 \mathrm{~W}$ animals when no significant difference in MAP between SHR-SP and WKY was observed. Further studies are needed to elucidate the reflex responses in SHR-SP at the very young stage.

Percent decreases in MAP by ACh were greater in SHR-SP than in WKY at 4, 7 and $9 \mathrm{~W}$. The enhancement of this effect in SHR-SP was most obvious at $9 \mathrm{~W}$. These results suggest that hypotensive responses with ACh in SHR-SP were enhanced compared with those in WKY, especially in older animals. However, it was recently reported that the percent decreases in MAP following ACh were attenuated in SHR. ${ }^{12)}$ In the present experiments, the vasodilating effect of ACh might have been responsible for the hypotensive responses because no negative chronotropic effect was observed. Several investigators showed that the endothelium-dependent vasorelaxation with ACh against serotonin or noradrenaline contraction was diminished in SHR-SP. ${ }^{\text {i)-7) }}$ However, vasorelaxation with $\mathrm{ACh}$ against endothelin contraction in SHR-SP was rcported to have been enhanced compared with those in WKY. ${ }^{22)}$ Our experiments may suggest that the vasodilation of physiologically regulated resistance vessels following $\mathrm{ACh}$ is enhanced in SHR-SP.

Intravenous injection of ADS caused transient decreases in HR followed by increases in SHR-SP and WKY. Thus, the hypotensive effect of ADS might be caused by both its vasodilating effect and a transient decrease in HR. The percent decreases in MAP caused by ADS were attenuated in SHR-SP compared 
with WKY at almost all ages. Transient decrease in HR in SHR-SP was attenuated at 7 and $9 \mathrm{~W}$. This attenuation may contribute to the attenuation of the hypotensive effect of ADS in SHR-SP. ADS causes bradycardia via the cardiac $\mathrm{A}_{1}-\mathrm{K}^{+}$type receptor and causes vasorelaxation via the vascular $\mathrm{A}_{2}$ receptor. ${ }^{23)}$ Recently, it was reported that vascular relaxation caused by ADS is also mediated by ATP-sensitive potassium channels. ${ }^{24,25)}$ Our findings may suggest that the activity of cardiac or vascular receptors for ADS is reduced in SHR-SP. In conscious SHR, intravenous infusion of ADS caused a greater fall in blood pressure and heart rate than in WKY. ${ }^{13)}$ Although the reason for this discrepancy is not clear, further studies of the relationship between the reactivity to ADS and hypertension are needed.

In summarizing our present data concerning pressor and hypotensive agents, the pressor effect of NA but not Ang II was enhanced and reflex bradycardia was diminished in SHR-SP. The hypotensive effect of ACh was enhanced, but that of ADS was attenuated in SHR-SP. Augmented or attenuated hypotensive responses to ACh or ADS in SHR-SP were contrary to the data reported in SHR. These discrepancies between SHR-SP and SHR still remain to be elucidated.

In conclusion, 1) the pressor effect of NA was enhanced, 2) bradycardias following pressor effects with NA or Ang II were attenuated, and 3) hypotensive and bradycardic responses of ADS were attenuated in the developing stages of hypertension in SHR-SP. Mechanisms underlying these phenomena might contribute to the development or maintenance of hypertension in SHR-SP.

\section{References}

1. Okamoto K, Yamori Y, Nagaoka A: Establishment of the stroke-prone spontaneously hypertensive rat (SHR). Circ Res 35 (suppl I): 1143, 1974

2. Berecek $\mathrm{KH}$, Schwertschlag U, Gross F: Alteration in renal vascular resistance and reactivity in spontaneous hypertension of rats. Am J Physiol 238: H287, 1980

3. Nagaoka A, Toyoda $S$, Iwatsuka $H$ : Increased renal vascular reactivity to norepinephrine in strokeprone spontaneously hypertensive rat (SHR). Life Sci 23: 1159, 1978

4. Nakata N, Abiko K, Ikawa H, Suzuki A: Responses to vasocontractile agents in the aortas and mesenteric arteries of M-SHRSP, SHRSP and WKY, with special reference to aging. Jpn Heart J 28: 587,1987

5. Tesfamariam B, Halpern W: Endothelium-dependent and endothelium-independent vasodilation in resistance arteries from hypertensive rats. Hypertension 11: 440, 1988

6. Miyata N, Tsuchida K, Tanaka M, Otomo S: Impairment of endothelium-dependent relaxation and changes in levels of cyclic GMP in carotid arteries from stroke-prone spontaneously hypertensive rats. J Pharm Pharmacol 42: 763, 1990

7. Shimamura K, Osugi S, Moriyama K, Sunano S: Impairment and protection of endothelium-dependent relaxation in aortae of various strains of spontaneously hypertensive rats. J Cardiovasc Pharmacol 17 (Suppl 3): \$133, 1991

8. Davy M, Heimburger M, Midol-Monnet M, Beslot F, Gohen $Y$ : Cardiovascular effects of drugs acting on the autonomous nervous system, in awake, normotensive and hypertensive rats. Arch Int 
Pharmacodyn 274: 31, 1985

9. Touw KB, Haywood JR, Shaffer RA, Brody MJ: Contribution of the sympathetic nervous system to vascular resistance in conscious young and adult spontaneously hypertensive rats. Hypertension 2: 408, 1980

10. Kubo T: Increased pressor responses to pressor agents in spontaneously hypertensive rats. Can J Physiol Pharmacol 57: 59, 1979

11. Chiu EKY, McNeill JR: The effect of prolonged infusion and withdrawal of angiotensin II in the spontaneously hypertensive rat. Can J Physiol Pharmacol 64: 748, 1986

12. Takata $Y$, Koga $T$, Kobayashi K, Takishita S, Fujishima M: Decrease in endothelium dependent hypotension in spontaneously hypertensive rats. Jpn Circ J 54: 183, 1990

13. Ohnishi A, Biaggioni I, Deray G, Branch RA, Jackson EK: Hemodynamic effects of adenosine in conscious hypertensive and normotensive rats. Hypertension B: 391, 1986

14. Masui M, Kawakami M, Nakajima M, Hara S, Ito H, Ueda M: Prophylactic effects of novel dihydropyrazolopyridine (83-0256) and dihydrothienopyridine (83-0312, 83-0327) calcium antagonists in stroke-prone spontaneously hypertensive rats. Drug Dev Res 20: 453, 1990

15. Asano M, Aoki K, Matsuda T: Reduced $\beta$-adrenoceptor interaction of norepinephrine enhance contraction in the femoral artery from spontaneously hypertensive rats. J Pharmacol Exp Ther 223: 207, 1982

16. McCubbin JW, Green JH, Page IH: Baroceptor function in chronic renal hypertension. Circ Res 4: 205, 1956

17. Angell-James JE: Characteristics of single aortic and right subclavian baroreceptor fiber activity in rabbits with chronic renal hypertension. Circ Res 32: 149, 1973

18. Sapru HN, Wang SC: Modification of aortic baroceptor resetting in the spontaneously hypertensive rat. Am J Physiol 230: 664, 1976

19. Struyker-Boudier HAJ, Evenwel RT, Smits JFM, Essen HV: Baroreflex sensitivity during the development of spontaneous hypertension in rats. Clin Sci 62: 589, 1982

20. Bristow JD, Honour AJ, Pickering GW, Sleight P, Smyth HS: Diminished baroreflex sensitivity in high blood pressure. Circulation 39: 48, 1969

21. Takeshita A, Tanaka S, Kuroiwa A, Nakamura M: Reduced baroreceptor sensitivity in borderline hypertension. Circulation 51: 738, 1975

22. Wu C-C, Bohr DF: Role of endothelium in the response to endothelin in hypertension. Hypertension 16: 677,1990

23. Olsson RA, Peason JD: Cardiovascular purinoceptors. Physiol Rev 70: 761, 1990

24. Daut J, Maier-Rudolph W, von Beckerath N, Mehrke G, Gunther K, Goedel-Meinen L: Hypoxic dilation of coronary arteries is mediated by ATP-sensitive potassium channels. Science 247: 1341, 1990

25. Aversano T, Ouyang P, Silverman H: Blockade of the ATP-sensitive potassium channel modulates reactive hyperemia in the canine coronary circulation. Circ Res 69: 618, 1991 